\title{
Temperature-dependent signals in STEM Electron Beam-Induced Current (EBIC) Imaging
}

William A. Hubbard ${ }^{1}$, Edward. R. White ${ }^{1}$, Matthew Mecklenburg ${ }^{2}$, and B. C. Regan ${ }^{1}$

1. Department of Physics \& Astronomy and California NanoSystems Institute, University of California, Los Angeles, CA, USA

2. Center for Electron Microscopy and Microanalysis, University of Southern California, Los Angeles, CA, USA

Transmission electron microscopy (TEM) has become an essential analysis tool as nanoscale phenomena have become central to current technology. While the most important dynamics in nanoscale devices are electronic or thermal, in conventional TEM techniques the imaging contrast is primarily determined by the physical structure of the sample. In other words, TEM images measure the coherent scattering (diffraction and phase contrast) and the incoherent scattering (diffraction, $\mathrm{Z}$, and thickness contrast) of primary beam electrons by the atomic nuclei. While such images are powerful nanoscale analysis tools, they typically only locate and, less frequently, chemically identify the sample's constituent atoms.

Electron beam-induced current (EBIC) imaging provides a probe of a sample's electronic structure that can be complementary to standard scanning TEM (STEM) imaging of a sample's physical structure. EBIC measures an electrical current generated in the sample by the electron beam: images are generated by connecting a current amplifier to a sample and mapping current as a function of the position of the STEM beam. While EBIC is traditionally deployed in a scanning electron microscope (SEM EBIC), STEM EBIC is different in two important ways: the electron beam energy is typically much higher, and the samples are electron-transparent. The former results in higher-energy secondary electrons which can more readily escape the sample [1], while the latter makes absorption negligible and improves the achievable spatial resolution [2]. In addition to electon-hole pair separation typically mapped in SEM EBIC, EBIC in STEM can also quantify secondary electron emission at the source. Previously STEM EBIC has primarily been used to study intrinsic electric fields in samples [2,3]. Here we discuss STEM EBIC's potential for application as an in situ temperature mapping technique with high spatial resolution.

Using standard microfabrication tools, we fabricated electron-transparent windows in $200 \mu$ m-thick silicon substrates. Over each window we placed a Pt heater electrode, a conformal layer of ALD alumina, and then Pt probe electrodes (see Figure 1). The alumina layer prevented current originating in the heater from reaching the probes attached to the current amplifier. Figure 1 shows three, simultaneously acquired, plan-view images of the heater/probe design: the first two are standard STEM images (bright field and annular dark field respectively), while the third is the STEM EBIC image. Here $140 \mu \mathrm{W}$ was dissipated in the heater on the left, while the probe electrodes on the right served as current collectors and were attached in parallel to the EBIC transimpedance amplifier. No special contrast variation appears in the standard STEM images, which are nearly indistinguishable from those acquired at zero heater power (data not shown). However, an obvious left-to-right signal gradient is evident in the EBIC image of the probes, matching the expected temperature profile of hot-to-cold moving away from the heater. Evidently the EBIC signal has a substantial component that is temperature-sensitive. 
Despite the alumina layer, the current measured by the transimpedance amplifier changes as a function of heater power, even when the beam is not incident on the sample (black plot in Figure 1). We subtract the electron emission and membrane background (measured in a region far from the heater) from the average signal in a region of interest (ROI, red box) in the EBIC image to arrive at the true, beamdependent EBIC signal (red plot in Figure 1). We attribute this signal, which is independent of the polarity of the heater bias, to thermal promotion of secondary electron emission during the beam interaction. A more quantitative understanding of this signal could yield an alternative method for temperature mapping in TEM.

\section{References:}

[1] MS Chung and TE, Journal of Applied Physics 45 (1974), p. 707-709.

[2] ER White, A Kerelsky, WA Hubbard et al, Applied Physics Letters 107 (2015), 223104.

[3] KL Bunker et al, MRS Proceedings 743 (2002), L1010.

[4] PR Emtage and W Tantraporn, Physical Review Letters 8 (1962), p. 267-268.

[5] This work has been supported by FAME, one of six centers of STARnet, a Semiconductor Research Corporation program sponsored by MARCO and DARPA, by National Science Foundation (NSF) award DMR-1611036, and by NSF STC award DMR-1548924. The authors acknowledge the use of instruments at the Electron Imaging Center for NanoMachines supported by NIH 1S10RR23057 and the CNSI at UCLA.
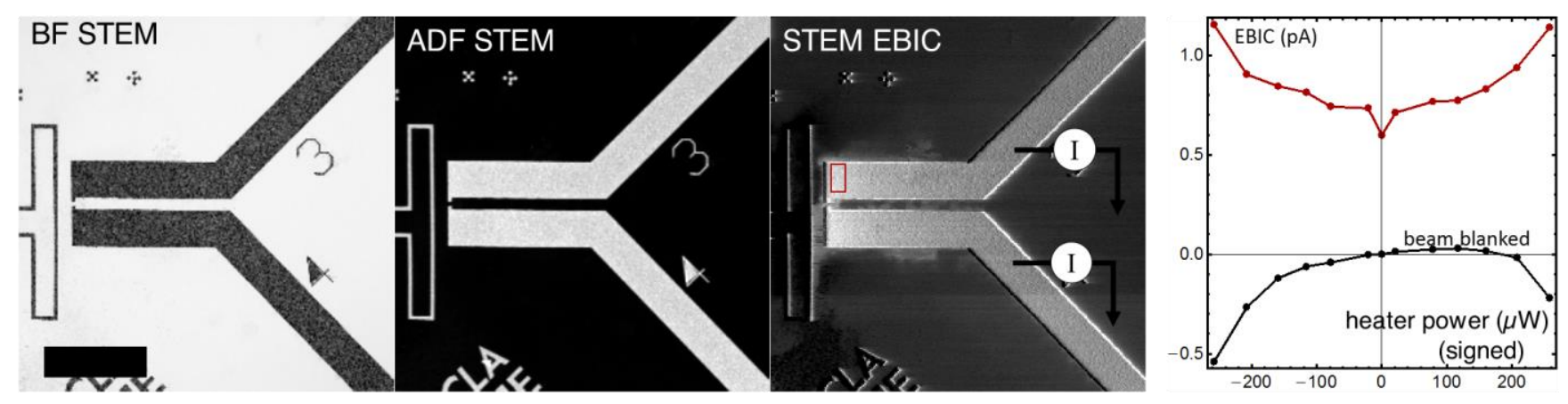

Figure 1. Bright-field and annular dark-field STEM and STEM EBIC images of a heating/biasing device. The images were simultaneously acquired while the Pt heater at the left of the image was dissipating $140 \mu \mathrm{W}$, and the Pt probe electrodes on the right were connected to the EBIC amplifier. The scale bar is $2 \mu \mathrm{m}$. The average EBIC signal measured in the ROI (red box) for a similar sample is plotted in red to the right. The black plot shows the beam-independent, heater-induced current, measured while the electron beam is blanked. 